\title{
First Antarctica light curve
}

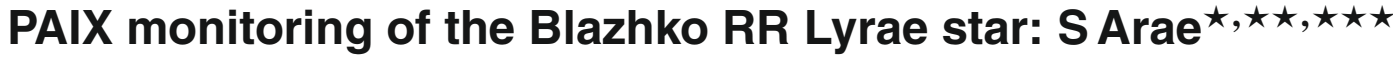

\author{
M. Chadid ${ }^{1,2}$, J. Vernin ${ }^{1}$, D. Mekarnia ${ }^{1}$, E. Chapellier ${ }^{1}$, H. Trinquet $^{1}$, and G. Bono ${ }^{3,4}$ \\ ${ }^{1}$ Observatoire de la Côte d'Azur, Université Nice Sophia-Antipolis, UMR 6525, Parc Valrose, 06108 Nice Cedex 02, France \\ e-mail: chadid@unice.fr \\ 2 Antarctica Reaserch Station, South Pole TAAF, Antarctica \\ 3 Dipartimento di Fisica, Universita di Roma Tor Vergata via Della Ricerca Scientifica 1, 00133 Roma, Italy \\ 4 INAF, Rome Astronomical Observatory, via Frascati 33, 00040 Monte Porzio Catone, Italy
}

Received 23 April 2010 / Accepted 1 June 2010

ABSTRACT

\begin{abstract}
Context. In the context of long and continuous time-series photometry and after the MOST, CoRoT, KEPLER space missions and large geographic longitude ground-based networks, a new method is offered by the polar location helping to cope with the problem associated with the Earth's day-night cycle.

Aims. We present the first long time -series photometry from Dome Charlie in the heart of Antarctica and analyze 4400 photometric measurements of RR Lyrae star S Arae obtained in July and August 2007.

Methods. The 40-cm telescope and Photometer AntarctIca eXtinction named PAIX were used for long photometric measurements with high time resolution and a duty cycle of $65 \%$. The Fourier technique was used for the frequency analysis. New high-precision spectroscopic observations of S Arae were also collected using HARPS over one pulsation cycle of S Arae.

Results. We confirm the known radial fundamental period of 0.452 day. The high order of its harmonics shows the nonlinear nature of the radial mode. The Blazhko modulation is directly detected in the spectrum and shows a value around $48 \mathrm{~d}$. Together with the Blazhko frequency we also detected triplet structure components up to 15th order showing a nonlinear behavior of the Blazhko modulation. Thus, we conclude that S Arae is a bona fide Blazhko star. The data reveal that there are irregular changes in the S Arae atmosphere during the Blazhko cycle. A residual scatter phenomenon is detected and is more intense where the nonlinear effects are strongest. Therefore, the connection between temporal hydrodynamic phenomena and such modulation has to be taken into account in Blazhko models.

Conclusions. High-precision CCD photometry with a very good time resolution can be undertaken at Dome Charlie in Antarctica, and it helps to complete astrophysical measurement time-series.
\end{abstract}

Key words. techniques: spectroscopic - methods: data analysis - techniques: photometric - shock waves - stars: variables: RR Lyrae - stars: atmospheres

\section{Introduction}

Asteroseismology is currently one of the fundamental techniques for improving our understanding of the internal structure of stars. On the observational side, progress is limited by the data accuracy needed to detect numerous modes with small amplitudes and by the discontinuous nature of typical groundbased data strings, which often introduces ambiguities in the determination of oscillation frequencies. Space missions such as MOST, CoRoT, and Kepler overcome quoted difficulties and significantly improved the scientific impact of asteroseismological investigations. Time series data with no gaps can be collected with ground-based telescopes at a polar site. For the first time we present a photometric data set with these features collected from Dome Charlie in Antarctica.

* Based on spectroscopic observations as well, obtained at the European Southern Observatory in Chile in the programs P79.D-0462.

$\star \star$ Tables 1-3 are only available in electronic form at

http: //www . aanda.org

$\star \star \star$ The table of the photometry is only available in electronic form at the CDS via anonymous ftp to

cdsarc.u-strasbg.fr $(130.79 .128 .5)$ or via

http://cdsweb.u-strasbg.fr/cgi-bin/qcat?J/A+A/516/L15
We have observed the RR Lyrae star S Arae, one of the largest amplitude RR lyrae stars, crossed by hypersonic shock waves (Chadid et al. 2008). In a recent investigation Wils \& Sodor (2005) suggests that S Arae might be a Blazhko RR Lyrae. This effect was discovered more than one century ago (Blazhko 1907), but we still lack a firm explanation of the physical mechanism(s) governing this intriguing phenomenon. This effect affects a large fraction of the RR Lyrae stars and shows a periodic modulation of their light curve amplitudes and phases on timescales of typically tens to hundreds of days. As discussed by Chadid \& Chapellier (2006) and Chadid et al. (2004), none of the currently suggested models - the resonance model the involves the non-radial modes (Dziembowski \& Mizerski 2004) and the magnetic oblique rotator (Shibahashi 2000) - is able to explain the complexity of the observed properties of the Blazhko modulation. Recently, Stothers $(2006,2010)$ has given a new interpretation of the modulating Blazhko effect, based on a convective turbulent dynamo inside the hydrogen and helium ionization zones, where the dynamo period is identical to the Blazhko period. The Stothers theory does not require any non-radial mode, however there has been no unambiguous proof of the nonradial mode presence in RR Lyrae stars so far. Nonradial modes have 
been identified by Chadid et al. (1999) using spectroscopic data of RR Lyr itself, by Gruberbauer et al. (2007) using photometric data collected with the MOST satellite of mixed mode pulsator AQ Leo and by Chadid et al. (2010) using time series data collected with CoRot of the fundamental RR Lyrae V1127 Aql.

These results are not enough to rule out the Stothers model, which still remains a qualitative interpretation of the modulation. They do, however, underline the importance of long and discontinuous data strings to address the Blazhko effect quantitatively.

We present and analyze the first data from PAIX Photometer AntarctIca eXtinction - obtained in July and August 2007. Our primary target is the RR ab star S Arae. In Sect. 2 we present the long time-series photometric polar observations with PAIX and describe the data reduction. Section 3 presents the data analysis and its results. In Sect. 4 we discuss our results in the framework of the understanding of S Arae pulsation and Blazhko effect. Finally, the conclusion and outlooks for future investigations are given in Sect. 5.

\section{Instrumentation, observations and data processing}

\subsection{PAIX}

PAIX - Photometer AntarctIca eXtinction - gives new insights in coping with the Blazhko effect challenge and is a great opportunity to benefit from access to one of the best astronomical sites on Earth - Dome Charlie - at a height of $3300 \mathrm{~m}$ on $75^{\circ} 06^{\prime} 04^{\prime \prime} \mathrm{S}$, $123^{\circ} 20^{\prime} 52^{\prime \prime} \mathrm{E}$ of the Antarctica plateau. During night time - polar winter - conditions, the seeing rises from 1 arcsec in March to peak around 2 arcsec in August and then decreases slowly (Vernin et al. 2009). Dome Charlie attracts strong interest from the time-series observation studies. Long, continuous, and quasiinterrupted observations could be carried out during one polar night with Earth's day-night duty cycle of $65 \%$.

PAIX is a $40 \mathrm{~cm}$ Ritchey-Chretien optical telescope, in combination with F/D ratio of 10 , located at Dome Charlie in the open field, without any shelter, and installed at ice level. The detector used is a KAF 400 CCD camera with $768 \times 512$ elements of $9 \mu \mathrm{m}$ size with a $22^{\prime} \times 14.5^{\prime}$ field of view, together with a focal reducer that increases the field of view by almost a factor of four, The camera is cooled with a Peltier assembly, without temperature regulation. The quantum efficiency is 3\%, 33\%, $40 \%$ and $8 \%$ at $400,550,700$, and $1000 \mathrm{~nm}$ respectively. PAIX was antarctized to run under conditions where the temperatures are as low as around $-81 \mathrm{deg}$. C. PAIX and the $40 \mathrm{~cm}$-telescope were designed and built by PaixTeam in Université Nice SophiaAntipolis and installed at Dome Charlie by one of the co-authors (Chadid).

\subsection{Antarctica observations and data reduction}

To illustrate the potential of PAIX photometry for RR Lyrae stars, we present the first results for the RR Lyrae Star, S Arae, with ( $\alpha=17^{\mathrm{h}} 59^{\mathrm{m}} 10^{\mathrm{s}} .73, \delta=-49^{\circ} 26^{\prime} 00^{\prime} \cdot 45$, J2000). The $V$ mag is around 10 and 11.5 and it has a pulsation period of 0.452 days. Optical photometric data were performed for $700 \mathrm{~h}$ (29 days) during 15 to 24 July and 10 to 30 August 2007, a total of $4400 \mathrm{CCD}$ frames were acquired with 40-60 s integration times, with a duty cycle of $65 \%$, high-precision optical photometric measurements and an average time resolution $\Delta \mathrm{T} / \mathrm{P}$ around $0.15 \%$ of the pulsation period, where $\Delta \mathrm{T}$ is the exposure time and $\mathrm{P}$ the pulsation period. Calibration frames were obtained at the end of the science observation and consisted of
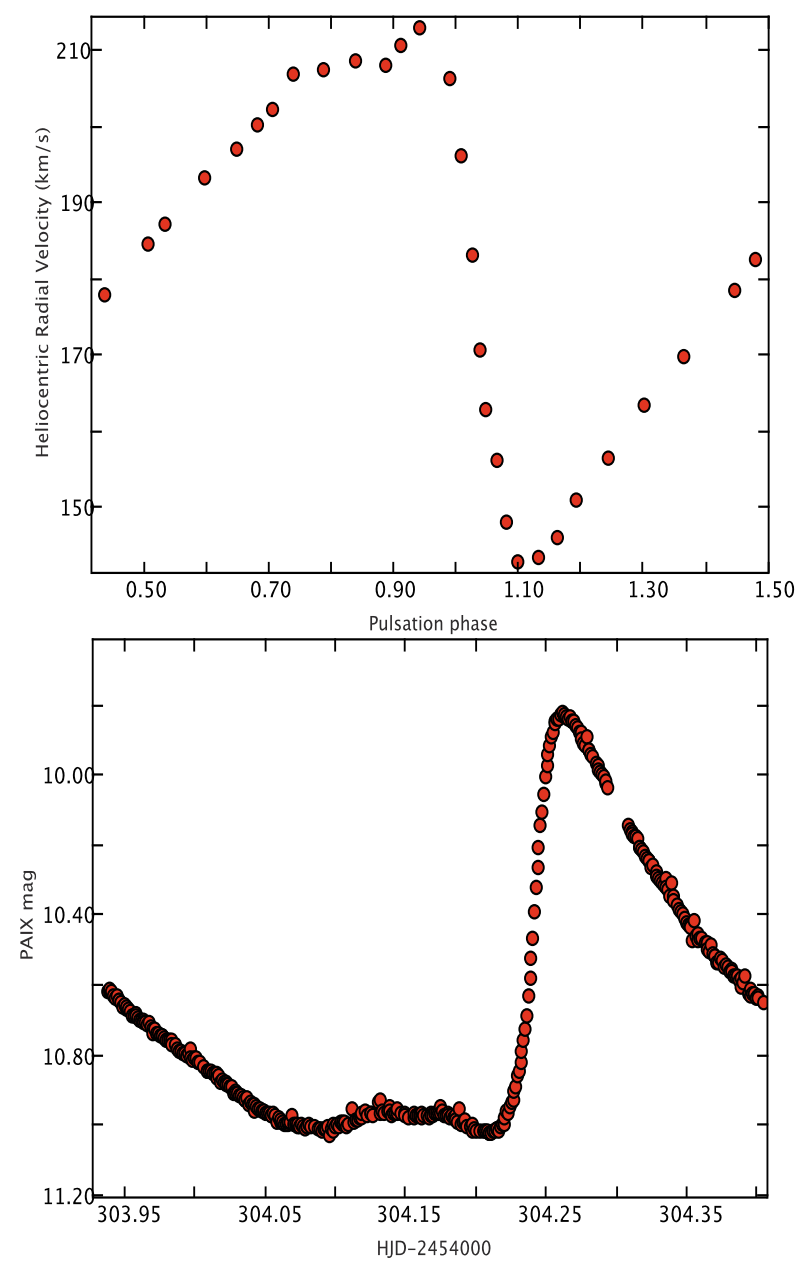

Fig. 1. HARPS correlation heliocentric radial velocity curve and PAIX light curve of S Arae.

5 twilight flat fields and a series of biases. The flat fields were done using the horizon pointing when the sun was below the horizon. Magnitudes were estimated for 4 standard stars identified in the NOMAD1(Zacharias et al. 2005) catalog as 04050770258, 086, 296, and 0405-0769976. Figure 1 (bottom) shows the PAIX light curve over one pulsation period of S Arae, revealing nonsinusoidal and highly nonlinear modulation.

\subsection{HARPS observations and data reduction}

Together with the PAIX light curve studies, we observed S Arae during one pulsation period, using the High-Accuracy Radial Velocity Planetary Searcher Spectrograph HARPS (Mayor et al. 2003). This high-resolution Echelle Spectrograph $(R=115000)$ is fiber-fed, cross-disperssed, by the ESO 3.6-m telescope at La Silla Observatory. We carried out, on 23 and 24 July, 2007, 29 measurements using the HARPS Classical fibre spectroscopic mode covering the wavelenght range from 380 to $690 \mathrm{~nm}$ with an exposure time around $10 \mathrm{mn}$ giving an average time resolution of $1.50 \%$ and a signal to noise ratio better than 50 per pixel at $550 \mathrm{~nm}$. The radial velocities (Table 1) were obtained with the standard HARPS reduction pipeline, based on the cross-correlation with a stellar template (Baranne et al. 1996). Almost all lines used are metallic and consequently the reduced radial velocities refer to the average photospheric motion. Figure 1 (top) shows the HARPS correlation radial velocity curve of S Arae with uncertainties better than $100 \mathrm{~m} \mathrm{~s}^{-1}$. 
Like PAIX light curve, the radial velocity curve shows a highly nonlinear behavior just before the maximum, i.e. pulsation phases ranging from $\phi \sim 0.75$ to 0.90 .

\section{Frequency analysis}

The PAIX data of S Arae provide 64 pulsation cycles. The heights of maxima and minima of the light curves clearly indicate a modulation period (Fig. 2 (top)) of the light curve amplitude and phase. The frequency analysis is carried out using the Period04 software (Lenz \& Breger 2005). The Fourier decomposition consists of fitting the magnitude measurements by means of the series

$m(t)=A_{0}+\sum_{i=1, N} A_{i} \sin \left[2 \pi F_{i}\left(t-T_{0}\right)+\Phi_{i}\right]$,

where $T_{0}$ is the initial epoch value of the data set, HJD $T_{0}=$ 2452691.91260.

The original spectrum is dominated by the main pulsation frequency $f_{1}=2.2127 \mathrm{~d}^{-1}$ and by its harmonics up to the 18 th order (Fig. 3). After prewhitening the spectrum with the main pulsation frequency and its harmonics, we clearly see the triplet frequencies that are a typical feature of Blazhko RR Lyrae stars (see Fig. 3 insert panel). The first side peak appears at $f_{1}-f_{\mathrm{b}}=$ $2.1921 \mathrm{~d}^{-1}$. This yields an initial value for the Blazhko frequency of $f_{\mathrm{b}}=0.0207 \mathrm{~d}^{-1}$ or a Blazhko period $P_{\mathrm{b}}=48 \mathrm{~d}$. Subsequent prewhitening yields additional triplet frequencies equally spaced around the main frequency and its harmonics. The triplet components are detected up to the $15 \mathrm{rd}$ order. The Blazhko frequency $f_{\mathrm{b}}$ itself is also detected directly from the data. Its detection implies that there is a variation in the mean brightness of the star with the Blazhko cycle. A third prewhitening of all modulation frequencies cited in Table 2 gave the residual spectrum shown in Fig. 3 (insert of insert panel). The critical point of where to stop in the frequency analyses is the signal-to-noise ratio sig $=5.5$ value which served as a controlling criterion. Table 3 summarizes amplitudes and phases resulting from a Fourier analysis during one pulsation cycle on the cross-correlation, heliocentric, radial velocity measurements listed in Table 1 . The results show that the heliocentric radial velocity is dominated by the main pulsation frequency $f_{1}=2.213 \mathrm{~d}^{-1}$ and its harmonics up to the 10th order.

\section{Discussion}

Frequency analysis of both radial velocity curve and light curve shows exponential by decreasing amplitudes of the harmonics of the main pulsation frequency respectively up to 10 th and 18 th harmonic component demonstrating a nonlinear havior of the main pulsation. S Arae has been listed recently as a suspected Blazhko star by Wils \& Sodor (2005) with a Blazhko period of 49.5 days. the direct detection of the Blazhko frequency itself from the data and the triplet structure components up to 15th order show that $\mathrm{S}$ Arae is a bona fide Blazhko star with a strong amplitude modulation and a Blazhko cycle around 48 days.

Figure 1 shows the correlation radial velocity curve and its reverse light curve taking at the same time and over one pulsation period of S Arae. Both reveal very significant elbow and bump respectively consequence of the secondary shock, early shock, as interpreted by Hill (1972) and Gillet \& Crowe (1988). We detected that the bump becomes more pronounced as the light curve amplitude decreases and vice versa. The descending branch and the ascending branch of the radial velocity curve and the light curve respectively occur during a very
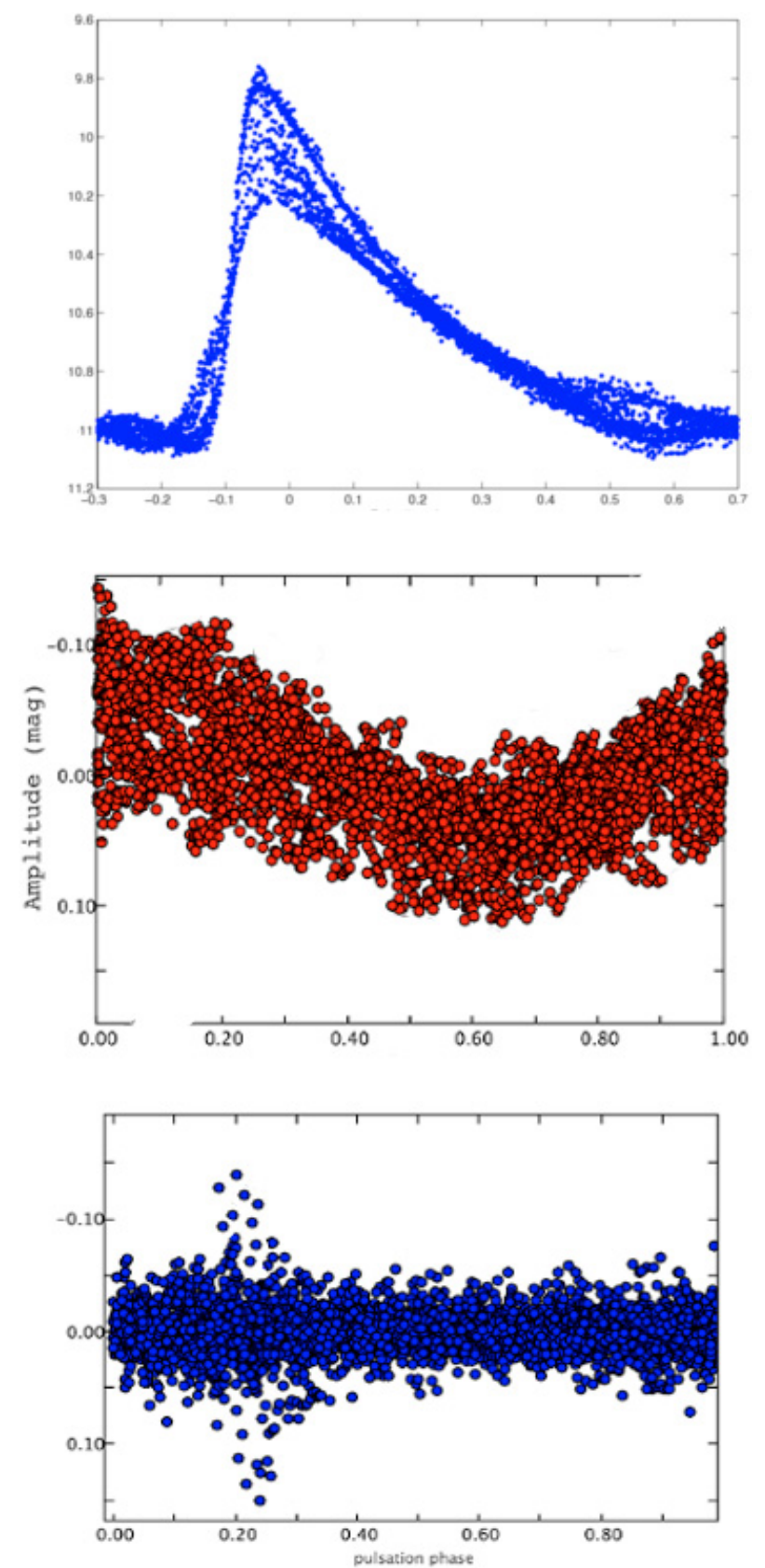

Fig. 2. Folded PAIX light curve of S Arae. Upper panel: folded with the main pulsation $P_{1}$. Middle panel: prewhitened with the main frequency $f_{1}$ and its harmonics, folded with $f_{1}-f_{\mathrm{b}}$. The lower panel: residual light curve after prewhitening with all frequencies listed in Table 2 , folded with $P_{1}$.

small phase interval, corresponding to $\sim 8 \%$ of the pulsation period and strongly associated with the hypersonic shock wave in the S Arae atmosphere as detected by Chadid et al. (2008). After prewhitening of the light curve with all frequencies listed in Table 2, we detected intense variations that only appear in a narrow phase interval of the pulsation cycle, $\sim 10 \%$ of the pulsation period. This residual scatter phenomenon occurs during the ascending branch of the light curve (see Fig. 2), centered at its maximum at phase of the middle of the ascending branch of the light curve. A similar residual phenomenon has already been shown by Chadid \& Chapellier (2006) from spectroscopic data of the Blazhko star RR Lyr itself and it is strongly connected to the passage of the main shock wave across the atmosphere. 


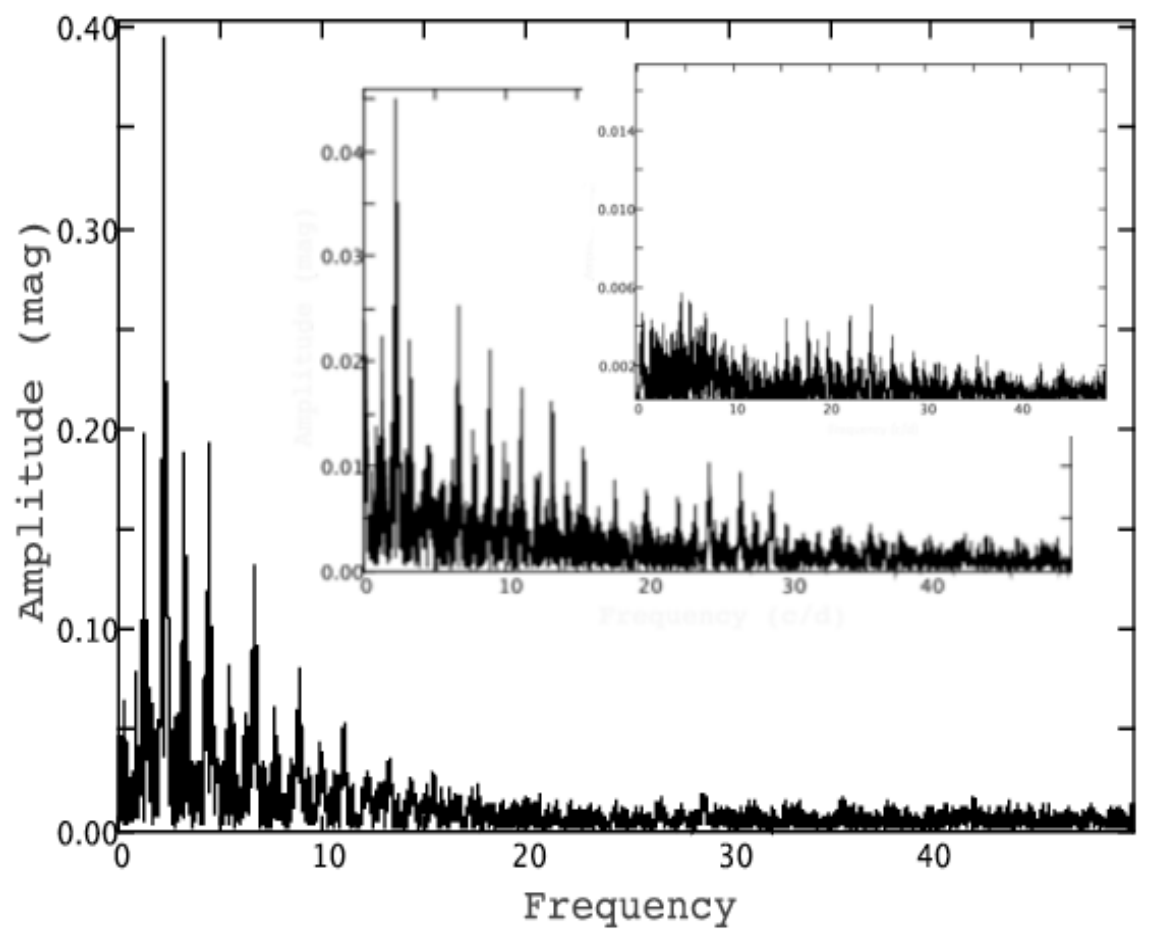

Fig. 3. Fourier spectra on the light curve of S Arae. The insert panels respectivly show the prewhitened spectrum with the main pulsation frequency and its harmonics and the residual spectrum when all frequencies listed in Table 2 have been removed.

This temporal hydrodynamical phenomenon introduce a large deviation in the magnitude measurements and then an irregular behavior of the Blazhko modulation. The phase interval of the residuals in RR Lyr itself is twice larger $(\sim 20 \%$ of the pulsation period) than in S Arae. This is puzzling evidence, since the atmosphere of this object is crossed by hypersonic shocks. The residual scatter becomes more intense as the nonlinear effects increases and vice versa. The connection between temporal hydrodynamical phenomenon and this modulation has to be taken into account in Blazhko models.

\section{Conclusions and outlooks}

We have presented and analyzed 700 continuous hours of optical photometric observations from the south polar site - Dome Charlie - in the heart of Antarctica, during the 2007 polar night with 40-60 s integration times and Earth's day-night duty cycle of $65 \%$. A total of 4400 CCD frames were acquired with the 40-cm PAIX telescope for the RR Lyrae star S Arae. We conclude that high-precision CCD photometry with a very good time resolution can be undertaken at Dome Charlie in Antarctica, even with the low-cost commercial components and under very harsh working conditions, and can help in completing astrophysical measurement time series. PAIX mission continues to explore the south polar sky and even could, in some favorable cases, challenge photometry from space.
Acknowledgements. It is a real pleasure to acknowledge the referee Christoffel Waelkens for the very positive comments that substantially improved the content of this article.

We thank the US Air Force Research Laboratory and Agence Nationale de la Recherche (ANR-05-BLAN-0033-01) for their great support and the Polar Institut Paul Emile Victor for the precious infrastructure help.

M. Chadid is indebted to all who participated in her Antarctica expeditions $\&$ the PAIX programs.

\section{References}

Baranne, A., Queloz, D., Mayor, M., et al. 1996, A\&A, 119, 373 Blazhko, S. 1907, Astr. Nachr, 175, 325

Chadid, M., \& Chapellier, E. 2006, A\&A, 456, 305

Chadid, M., Kolenberg, K., Aerts, C., \& Gillet, D. 1999, A\&A, 352, 201

Chadid, M., Wade, G. A., Shorlin, S. L. S., \& Landstreet, J. D. 2004, A\&A, 413, 1087

Chadid, M., Vernin, J., \& Fossat, E. 2006, EAS, 14, 281

Chadid, M., Vernin, J., \& Gillet, D. 2008, A\&A, 491, 537

Chadid, M., Benko, J. M., Szabo, R., et al. 2010, A\&A, 510, 39

Dziembowski, W. A., \& Mizerski, T. 2004, Acta Astron., 54, 363

Gillet, D., \& Crowe, R. A. 1988, A\&A, 199, 242

Hill, S. J. 1972, ApJ, 178, 793

Lenz, P., \& Breger, M. 2005, CoAst., 146, 53

Mayor, M., Pepe, F., Queloz, D., et al. 2003, Msngr, 114, 20

Shibahashi, H. 2000, ASP Conf. Ser. 203, 299

Stothers, R. B. 2006, ApJ, 652, 643

Stothers, R. B. 2010, PASP, 122, 536

Vernin, J., Chadid, M., Aristidi, E., et al. 2009, A\&A, 500, 1271

Wils, P., \& Sodor, A. 2005, IBVS, 5655 
M. Chadid et al.: First Antarctica light curve

Table 1. Journal of HARPS spectroscopic observations of S Arae.

\begin{tabular}{lrr}
\hline \hline Date & $\begin{array}{r}\text { HJD } \\
2400000+\end{array}$ & $\begin{array}{r}\text { HRV } \\
\mathrm{km} \mathrm{s}^{-}\end{array}$ \\
\hline 2007 Jul. 23 & 54304.4475042 & 200.07842 \\
2007 Jul. 23 & 54304.4589157 & 202.06640 \\
2007 Jul. 23 & 54304.4742391 & 206.83291 \\
2007 Jul. 23 & 54304.4960206 & 207.37144 \\
2007 Jul. 23 & 54304.5177210 & 208.70000 \\
2007 Jul. 23 & 54304.5403705 & 207.98420 \\
2007 Jul. 23 & 54304.5512434 & 210.55950 \\
2007 Jul. 23 & 54304.5644435 & 212.84540 \\
2007 Jul. 23 & 54304.5884586 & 206.35990 \\
2007 Jul. 23 & 54304.6021365 & 183.03880 \\
2007 Jul. 23 & 54304.6099215 & 170.61383 \\
2007 Jul. 23 & 54304.6127631 & 162.72694 \\
2007 Jul. 23 & 54304.6386763 & 142.82990 \\
2007 Jul. 23 & 54304.6511873 & 143.29410 \\
2007 Jul. 23 & 54304.6648673 & 145.97919 \\
2007 Jul. 23 & 54304.6789176 & 150.88562 \\
2007 Jul. 23 & 54304.7007453 & 156.48577 \\
2007 Jul. 23 & 54304.7198754 & 163.39000 \\
2007 Jul. 23 & 54304.7202532 & 169.74697 \\
2007 Jul. 23 & 54304.7926973 & 178.56295 \\
2007 Jul. 23 & 54304.8084720 & 182.62221 \\
2007 Jul. 24 & 54305.4457810 & 150.55950 \\
2007 Jul. 24 & 54305.5249907 & 155.99200 \\
2007 Jul. 24 & 54305.6249907 & 166.04168 \\
2007 Jul. 24 & 54305.6930155 & 177.88600 \\
2007 Jul. 24 & 54305.7239168 & 184.63600 \\
2007 Jul. 24 & 54305.7365088 & 187.22970 \\
2007 Jul. 24 & 54305.7465489 & 193.20310 \\
2007 Jul.24 & 54305.7655816 & 196.90850 \\
\hline
\end{tabular}

Table 2. Fourier amplitudes, phases and identification(s) for the PAIX data of the star S Arae.

\begin{tabular}{rrrr}
\hline \hline ID & $\begin{array}{r}\text { Frequency } \\
\text { c/d }\end{array}$ & $\begin{array}{r}\text { Amplitude } \\
\text { mag }\end{array}$ & $\begin{array}{c}\text { Phase } \\
\text { cycles }\end{array}$ \\
\hline Main frequency and harmonics \\
$f_{1}$ & 2.2127 & 0.3786 & 0.3944 \\
$2 f_{1}$ & 4.4254 & 0.1762 & 0.0429 \\
$3 f_{1}$ & 6.6393 & 0.1171 & 0.4139 \\
$4 f_{1}$ & 8.8510 & 0.0742 & 0.2472 \\
$5 f_{1}$ & 11.0640 & 0.0477 & 0.8300 \\
$6 f_{1}$ & 13.2772 & 0.0325 & 0.2515 \\
$7 f_{1}$ & 15.4891 & 0.0262 & 0.7871 \\
$8 f_{1}$ & 17.7019 & 0.0225 & 0.3477 \\
$9 f_{1}$ & 19.9194 & 0.0106 & 0.9005 \\
$10 f_{1}$ & 22.1304 & 0.0095 & 0.2901 \\
$14 f_{1}$ & 30.9774 & 0.0052 & 0.1188 \\
$16 f_{1}$ & 35.4040 & 0.0038 & 0.8746 \\
$17 f_{1}$ & 37.6174 & 0.0034 & 0.0080 \\
$18 f_{1}$ & 39.8299 & 0.0030 & 0.5157 \\
\hline Blazhko modulation frequency \\
$f_{\mathrm{b}}$ & 0.0206 & 0.02038 & 0.5308 \\
\hline Modulation triplet frequencies \\
$f_{1}-f_{\mathrm{b}}$ & 2.1921 & 0.0482 & 0.3745 \\
$2 f_{1}+f_{\mathrm{b}}$ & 4.4475 & 0.0216 & 0.2109 \\
$3 f_{1}+f_{\mathrm{b}}$ & 6.6598 & 0.0312 & 0.3859 \\
$4 f_{1}+f_{\mathrm{b}}$ & 8.8709 & 0.0241 & 0.2574 \\
$5 f_{1}+f_{\mathrm{b}}$ & 11.0833 & 0.0213 & 0.0969 \\
$6 f_{1}+f_{\mathrm{b}}$ & 13.2969 & 0.0189 & 0.4058 \\
$7 f_{1}-f_{\mathrm{b}}$ & 15.4701 & 0.0158 & 0.8672 \\
$8 f_{1}-f_{\mathrm{b}}$ & 17.6824 & 0.0148 & 0.9526 \\
$9 f_{1}+f_{\mathrm{b}}$ & 19.9441 & 0.0147 & 0.8734 \\
$10 f_{1}+f_{\mathrm{b}}$ & 22.1577 & 0.0127 & 0.5005 \\
$11 f_{1}+f_{\mathrm{b}}$ & 24.3713 & 0.0100 & 0.9729 \\
$12 f_{1}+f_{\mathrm{b}}$ & 26.5837 & 0.0081 & 0.7888 \\
$13 f_{1}+f_{\mathrm{b}}$ & 28.7973 & 0.0068 & 0.2861 \\
$15 f_{1}+f_{\mathrm{b}}$ & 33.2218 & 0.0047 & 0.8947 \\
\hline \multicolumn{4}{c}{}
\end{tabular}

Table 3. Fourier amplitudes, phases and identification(s) for the HARPS data of the star S Arae.

\begin{tabular}{rrrr}
\hline \hline ID & $\begin{array}{r}\text { Frequency } \\
\text { c/d }\end{array}$ & $\begin{array}{r}\text { Amplitude } \\
\mathrm{km} \mathrm{s}^{-1}\end{array}$ & $\begin{array}{r}\text { Phase } \\
\text { cycles }\end{array}$ \\
\hline$f_{1}$ & 2.213 & 21.005307 & 0.622751 \\
$2 f_{1}$ & 4.426 & 9.449569 & 0.659238 \\
$3 f_{1}$ & 6.639 & 4.677143 & 0.722872 \\
$4 f_{1}$ & 8.852 & 3.490279 & 0.811035 \\
$5 f_{1}$ & 11.065 & 2.421294 & 0.909750 \\
$6 f_{1}$ & 13.280 & 1.594122 & 0.979644 \\
$7 f_{1}$ & 15.490 & 0.514471 & 0.019409 \\
$8 f_{1}$ & 17.705 & 0.434692 & 0.304560 \\
$9 f_{1}$ & 19.920 & 0.330916 & 0.353024 \\
$10 f_{1}$ & 22.130 & 0.300264 & 0.378744 \\
\hline
\end{tabular}

